\title{
SHORTEST PATH SIMULATION USING INTERACTIVE SVG MAP
}

\author{
JONATHAN SIDI, LIEW WEI FA, SYAHRUL N. JUNAINI \\ Faculty of Computer Science and Information Technology, Universiti Malaysia Sarawak (UNIMAS) \\ 94300 Kota Samarahan, Sarawak, Malaysia
}

\begin{abstract}
This paper presents reports a design and development of the interactive map using Scalable Vector Graphics (SVG) that can be used to find the shortest path. Using our interactive map, the user will be able to set the route or path to the desired location. If that path is congested, the map will find the second shortest path to the user. In the usability study, we have tested the map with the mean value of 4.76 out of 5 .
\end{abstract}

\section{Introduction}

The main objective of our interactive map is to enable tourists to get familiar with Kuching Waterfront. Besides that, it provides an interactive SVG Map for tourist as a guide. It will guide tourist to their desire destination in waterfront using interactive SVG Map by showing shortest path inside the SVG Map. SVG is a language for describing two-dimensional graphics and graphical applications in XML.

Besides that, our proposed interactive SVG map is able to find the shortest path of a route. There are several existing algorithm that can find shortest path. The shortest path problem is vital to various applications including routing [1][5]. However, the problem of computing the shortest paths data has acquired little attention in the literature [2]. We have studied various algorithms such as $A^{*}$ Pathfinding Algorithm, Euclidean shortest path algorithm and Single source shortest path algorithm (Dijkstra's algorithm) are studied. The most suitable algorithm was used in our interactive SVG map.

The rest of this paper is organized as follows. Section 2 discusses related application of SVG in map and several related algorithm. Section 3 reports the design and development process of SMD translation tool. Finally, section 4 concludes the paper and highlights further work.

\section{Related Work}

\subsection{Putrajaya Interactive Map}

Putrajaya interactive map is an interactive map that displays public transport, public service, commercial building, government building, educational building, places of interest, and parks in Putrajaya, Malaysia. The user can interact with the map by selecting different categories by selecting the check box at the panel right side of the map and tabs located at the top of the map. If the user wants to view all the government buildings, he or she have to select the government building tab at the top of the map, Then the map with all the government building labeled will be shown. Besides that, the map also provides zoom-in and zoom-out function. This enables user zoom-in and zoom-out the map to have a clearer view of the location. This is the special function of this map which provides high interactivity with user by let the user draw a rectangular to zoom-in and zoomout the map. Figure 1 below shows the interactive map of the Putrajaya.

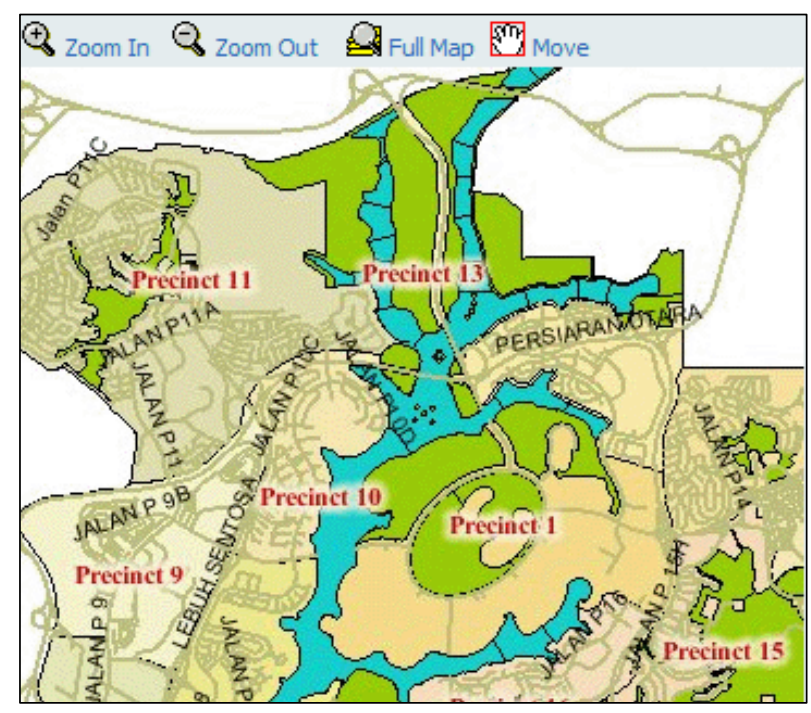

Figure 1. Putrajaya interactive map (copyright www.ppj.gov.my). 